\title{
Towards a consistent approach to nuclear structure: EFT of two- and many-body forces
}

\author{
R. Machleidt $\dagger$ and D. R. Entem $\ddagger$ \\ $\dagger$ Department of Physics, University of Idaho, Moscow, Idaho 83844, U. S. A. \\ $\ddagger$ Nuclear Physics Group, University of Salamanca, E-37008 Salamanca, Spain
}

\begin{abstract}
We review the nuclear forces currently in use, i. e., the high-precision NN potentials of the 1990's and the nuclear two- and many-body forces based upon chiral effective field theory (EFT). We argue that the EFT approach is superior to any of the older schemes. Since accurate chiral forces are available now, the stage is set for microscopic nuclear struture to move into a new and exciting era.
\end{abstract}

\section{Introduction}

This workshop deals essentially with microscopic nuclear structure which has the goal to derive the properties of atomic nuclei from the 'elementary' forces between nucleons. Thus, the input for microscopic nuclear structure calculations are the 'basic' nuclear forces: two-nucleon forces $(2 \mathrm{NF})$, three-nucleon forces $(3 \mathrm{NF}), \ldots$ It is the purpose of this contribution to review the nuclear forces that are presently in use. We can distinguish between two groups of current forces: the so-called high-precision nucleonnucleon (NN) potentials that were developed in the previous decade and NN potentials (plus associated 3NFs) based upon chiral perturbation theory. Presently, the majority of nuclear structure calculations are still conducted with high-precision potentials, but the interest in the chiral forces is increasing. The future belongs to the chiral approach for reasons that we will explain in sect. 3 .

Diversity has always been a characteristic feature of the NN potential market. Therefore, it is not possible to squeeze all current potentials into the above two categories. Examples for such exceptional cases are the Moscow potential [1, the highly nonlocal potentials developed by Doleschall and coworkers [2], and the NN potentials from inverse scattering by Shirokov et al. 3. All these potentials have recently been applied in nuclear structure yielding remarkable results. Unfortunately, because of lack of space, I cannot further elaborate on them.

\section{Historical perspective: The high-precision NN potentials}

In 1993, the Nijmegen group 4] published a phase shift analysis which described the $\mathrm{NN}$ data below $350 \mathrm{MeV}$ laboratory energy available at the time with the 'perfect' $\chi^{2}$ /datum of 1.0. This raised the expectation that also NN potentials should reproduce the NN data with similar precision. Note that even the best NN models of the 1980's, like the Paris [5] and the Bonn [6] potentials, fit the NN data typically with a $\chi^{2} /$ datum $\approx 2$ or more. To put microscopic nuclear structure theory to a reliable test, one needs 
Table 1. $\chi^{2}$ /datum for the reproduction of the 1992 and 1999 NN databases below $350 \mathrm{MeV}$ by the Nijmegen phase shift analysis 4 and two high-precision potentials: the CD-Bonn potential $\left[10\right.$ and the Argonne $V_{18}$ potential 8 .

\begin{tabular}{lccc}
\hline & $\begin{array}{c}\text { CD-Bonn } \\
\text { potential }\end{array}$ & $\begin{array}{c}\text { Nijmegen } \\
\text { PSA }\end{array}$ & $\begin{array}{c}\text { Argonne } \\
V_{18} \text { pot. }\end{array}$ \\
\hline \multicolumn{5}{c}{ proton-proton data } \\
$1992 p p$ database (1787 data) & 1.00 & 1.00 & 1.10 \\
After-1992 pp data (1145 data) & 1.03 & 1.24 & 1.74 \\
$1999 p p$ database (2932 data) & 1.01 & 1.09 & 1.35 \\
\hline \multicolumn{5}{c}{ neutron-proton data } \\
$1992 n p$ database (2514 data) & 1.03 \\
After-1992 $n p$ data (544 data) & 0.99 & 0.99 & 1.08 \\
$1999 n p$ database (3058 data) & 1.02 & 0.99 & 1.02 \\
\hline \multicolumn{5}{c}{ and } & $\boldsymbol{n p}$ data \\
$1992 N N$ database (4301 data) & 1.02 & 0.99 & 1.07 \\
$1999 N N$ database (5990 data) & 1.02 & 1.04 & 1.21 \\
\hline
\end{tabular}

a perfect $\mathrm{NN}$ potential such that discrepancies in the predictions cannot be blamed on a bad fit of the NN data.

So, the Nijmegen analysis triggered a feverish activity among groups traditionally involved in potential construction. The output was a new family/generation of NN potentials which eventually were dubbed the high-precision potentials. The research groups involved and the names of their new creations are, in chronological order:

- Nijmegen group [7]: Nijm-I, Nijm-II, and Reid93 potentials.

- Argonne group [8]: $V_{18}$ potential.

- Bonn group 9, 10: CD-Bonn potential.

All these potentials have in common that they are charge-dependent which is crucial to obtain a good fit of the $p p$ and $n p$ data. Moreover, they all need about 40-50 parameters to reproduce the 1992 Nijmegen $N N$ data base with a $\chi^{2} /$ datum $\approx 1$. Note, however, that since 1993 the $p p$ database has substantially expanded and for the current database the $\chi^{2} /$ datum produced by some of these potentials is not so perfect anymore (cf. table 1).

Concerning the theoretical basis of these potential, one could say that they are all-more or less - constructed 'in the spirit of meson theory' (e.g., all potentials include the one-pion-exchange (OPE) contribution). However, there are considerable differences in the details leading to considerable off-shell differences among the potentials.

The CD-Bonn potential uses the full, original, nonlocal Feynman amplitude for OPE, while all other potentials apply local approximations. As a consequence, the CDBonn potential has a weaker tensor force as compared to all other potentials. This is reflected in the predicted D-state probabilities of the deuteron, $P_{D}$, which is a measure of the strength of the nuclear tensor force. While CD-Bonn predicts $P_{D}=4.85 \%$, the other potentials yield $P_{D}=5.7(1) \%$. These differences in the strength of the tensor force lead to considerable differences in nuclear structure predictions. The CD-Bonn potentials predicts $8.00 \mathrm{MeV}$ for the triton binding energy, while the local potentials predict only $7.62 \mathrm{MeV}$. We note that the recent highly nonlocal Doleschall potential 2 predicts $P_{D}=3.6 \%$ and reproduces the entire triton binding of $8.48 \mathrm{MeV}$ from the $2 \mathrm{NF}$ alone. 
The OPE contribution to the nuclear force essentially takes care of the longrange interaction and the tensor force. In addition to this, all models must describe the intermediate and short range interaction, for which very different approaches are taken. The CD-Bonn includes (besides the pion) the vector mesons $\rho(769)$ and $\omega(783)$, and two scalar-isoscalar bosons, $\sigma$, using the full, nonlocal Feynman amplitudes for their exchanges. Thus, all components of the CD-Bonn are nonlocal and the off-shell behavior is the original one as determined from relativistic field theory.

The models Nijm-I and Nijm-II are based upon the Nijmegen78 potential [1] which is constructed from approximate one-boson-exchange (OBE) amplitudes. Whereas Nijm-II uses the local approximations for all OBE contributions, Nijm-I keeps some nonlocal terms in the central force component (but the Nijm-I tensor force is local). However, nonlocalities in the central force have only a very moderate impact on nuclear structure. If one wants to retain nonlocality, it is more important to keep the tensor force nonlocalities.

The Reid93 [7 and Argonne $V_{18} \quad 8$ potentials do not use meson-exchange for intermediate and short range; instead, a phenomenological parametrization is chosen. The Argonne $V_{18}$ uses local functions of Woods-Saxon type, while Reid93 applies local Yukawa functions of multiples of the pion mass, similar to the original Reid potential of 1968 [12]. At very short distances, the potentials are regularized either by exponential ( $V_{18}$, Nijm-I, Nijm-II) or by dipole (Reid93) form factors, which are all local functions.

Over the past ten years, the family of high-precision potentials has provided a useful service to the community. Practitioners in the field of microscopic nuclear structure and exact few-body calculations could finally be sure that their predictions, good or bad, had nothing to do with a bad fit of the NN data. Moreover, since all potentials reproduce the $\mathrm{NN}$ data base with the same $\chi^{2} /$ datum $\approx 1$ ('phaseequivalent' potentials), the impact of off-shell differences between potentials on nuclear structure predictions could be studied systematically and reliably [13, 14, 15, 16.

However, in spite of these great practical achievements, the high-precision potentials cannot be the end of the story. From a fundamental point of view, these potentials are not satisfactory at all. To achieve the acclaimed accuracy, the potentials use about 45 parameters. The underlying one-boson-exchange model has only about a dozen parameters [17. Additional parameters are introduced, e.g., by using partial wave dependent coupling constants, which is hard to justify by the underlying model. The motto is simply:

If you want more more accuracy, you have to use less theory.

This is a slap in the face of theoretical physics. Moreover, fundamental questions are unanswered, like:

- What is the connection to the fundamental theory of strong interactions, QCD?

- Why is the $2 \mathrm{NF}$ so much stronger than the $3 \mathrm{NF}$ ?

In the next section, we will show how to overcome these problems.

\section{The EFT approach to nuclear forces}

Quantum chromodynamics (QCD), the generally accepted theory of strong interactions, is non-perturbative in the low-energy regime characteristic for nuclear physics. For many years, this fact was perceived as the great obstacle for a derivation 
of the nuclear force from QCD - impossible to overcome except by lattice QCD. The effective field theory (EFT) concept has shown the way out of this dilemma. Notice that the QCD Lagrangian for massless up and down quarks is chirally symmetric, i. e., it is invariant under global flavor $S U(2)_{L} \times S U(2)_{R}$ equivalent to $S U(2)_{V} \times S U(2)_{A}$ (vector and axial vector) transformations. The axial symmetry is spontaneously broken as evidenced in the absence of parity doublets in the low-mass hadron spectrum. This implies the existence of three massless Goldstone bosons which are identified with the three pions $\left(\pi^{ \pm}, \pi^{0}\right)$. The non-zero, but small, pion mass is a consequence of the fact that the up and down quark masses are not exactly zero (some small, but explicit symmetry breaking). Thus, we arrive at a low-energy scenario that consists of pions and nucleons interacting via a force governed by spontaneously broken approximate chiral symmetry.

The effective Lagrangian describing this scenario is given by an infinite series of terms with increasing number of derivatives and/or nucleon fields, with the dependence of each term on the pion field prescribed by the rules of broken chiral symmetry. Applying this Lagrangian to NN scattering generates an unlimited number of Feynman diagrams. However, Weinberg showed [18] that a systematic expansion exists in terms of $\left(Q / \Lambda_{\chi}\right)^{\nu}$, where $Q$ denotes a momentum or pion mass, $\Lambda_{\chi} \approx 1 \mathrm{GeV}$ is the chiral symmetry breaking scale, and $\nu \geq 0$ (cf. figure 1). This has become known as chiral perturbation theory $(\chi \mathrm{PT})$. For a given order $\nu$, the number of contributing terms is finite and calculable; these terms are uniquely defined and the prediction at each order is model-independent. By going to higher orders, the amplitude can be calculated to any desired accuracy. Thus, the motto is now:

If you want more more accuracy, you have to use more theory (more orders).

This sounds more like what we expect from theoretical physics. Moreover, we have connected with QCD.

Following the first initiative by Weinberg [18], pioneering work was performed by Ordóñez, Ray, and van Kolck [19, 20] who constructed a NN potential in coordinate space based upon $\chi \mathrm{PT}$ at next-to-next-to-leading order (NNLO; $\nu=3$ ). The results were encouraging and many researchers became attracted to the new field. Kaiser, Brockmann, and Weise 21] presented the first model-independent prediction for the NN amplitudes of peripheral partial waves at NNLO. Epelbaum et al. 22] developed the first momentum-space NN potential at NNLO, and Entem and Machleidt 23. presented the first potential at $\mathrm{N}^{3} \mathrm{LO}$.

In $\chi \mathrm{PT}$, the NN amplitude is uniquely determined by two classes of contributions: contact terms and pion-exchange diagrams. There are two contacts of order $Q^{0}$ $\left[\mathcal{O}\left(Q^{0}\right)\right]$ represented by the four-nucleon graph with a small-dot vertex shown in the first row of figure 1. The corresponding graph in the second row, four nucleon legs and a solid square, represent the seven contact terms of $\mathcal{O}\left(Q^{2}\right)$. Finally, at $\mathcal{O}\left(Q^{4}\right)$, we have 15 contact contributions represented by a four-nucleon graph with a solid diamond.

Now, turning to the pion contributions: At leading order $\left[\mathrm{LO}, \mathcal{O}\left(Q^{0}\right), \nu=0\right]$, there is only the wellknown static one-pion exchange (OPE), second diagram in the first row of figure 1. Two-pion exchange (TPE) starts at next-to-leading order (NLO, $\nu=2$ ) and all diagrams of this leading-order two-pion exchange are shown. Further TPE contributions occur in any higher order. Of this sub-leading TPE, we show only two representative diagrams at NNLO and three diagrams at $\mathrm{N}^{3} \mathrm{LO}$. While TPE at NNLO was known for a while [19, 21, 22, TPE at $\mathrm{N}^{3} \mathrm{LO}$ has been calculated only 


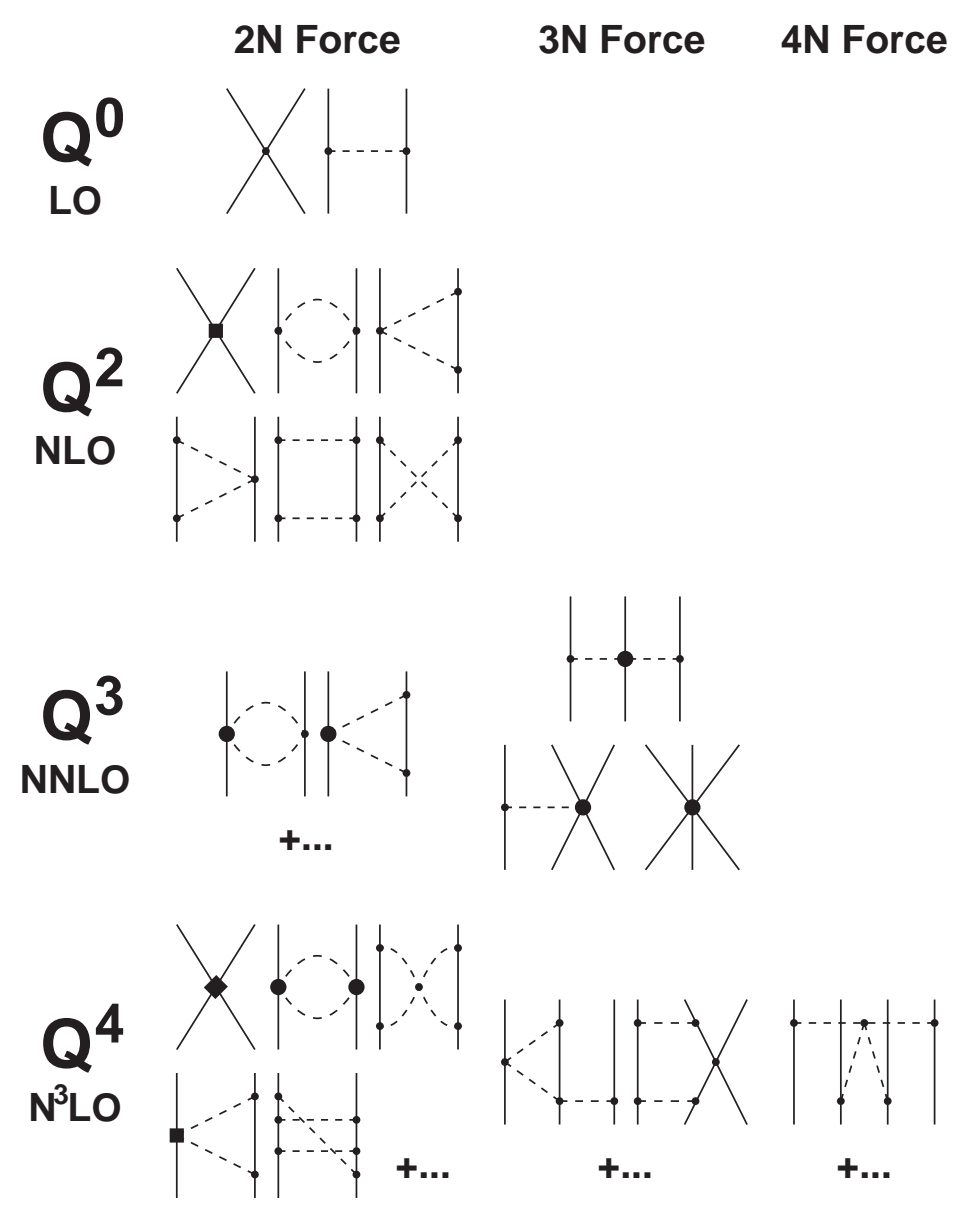

Figure 1. Hierarchy of nuclear forces in $\chi \mathrm{PT}$. Solid lines represent nucleons and dashed lines pions. Further explanations are given in the text.

recently by Kaiser 24]. All $2 \pi$ exchange diagrams/contributions up to $\mathrm{N}^{3} \mathrm{LO}$ are summarized in a pedagogical and systematic fashion in Ref. [25] where the modelindependent results for $\mathrm{NN}$ scattering in peripheral partial waves are also shown.

Finally, there is also three-pion exchange, which shows up for the first time at $\mathrm{N}^{3} \mathrm{LO}$ (two loops; one representative $3 \pi$ diagram is included in figure 1). In Ref. 26], it was demonstrated that the $3 \pi$ contribution at this order is negligible.

One important advantage of $\chi \mathrm{PT}$ is that it makes specific predictions also for many-body forces. For a given order of $\chi \mathrm{PT}, 2 \mathrm{NF}, 3 \mathrm{NF}, \ldots$ are generated on the same footing (cf. figure 1). At LO, there are no $3 \mathrm{NF}$, and at next-to-leading order (NLO), all 3NF terms cancel [18, 27. However, at NNLO and higher orders, welldefined, nonvanishing $3 \mathrm{NF}$ occur [27, 28]. Since $3 \mathrm{NF}$ show up for the first time at NNLO, they are weak. Four-nucleon forces (4NF) occur first at $\mathrm{N}^{3} \mathrm{LO}$ and, therefore, they are even weaker. 
Table 2. Number of parameters used for the $n p$ potential in various approaches discussed in the text.

\begin{tabular}{ccccc}
\hline & $\begin{array}{c}\text { Nijmegen } \\
\text { PWA93 }\end{array}$ & $\begin{array}{c}\text { CD-Bonn } \\
\text { "high } \\
\text { precision" }\end{array}$ & $\begin{array}{c}\text { NLO } \\
Q^{2} \\
\text { (NNLO) }\end{array}$ & $\begin{array}{c}\mathrm{N}^{3} \mathrm{LO} \\
Q^{4}\end{array}$ \\
\hline${ }^{1} S_{0}$ & 3 & 4 & 2 & 4 \\
${ }^{3} S_{1}$ & 3 & 4 & 2 & 4 \\
\hline${ }^{3} S_{1^{-}}{ }^{3} D_{1}$ & 2 & 2 & 1 & 3 \\
\hline${ }^{1} P_{1}$ & 3 & 3 & 1 & 2 \\
${ }^{3} P_{0}$ & 3 & 2 & 1 & 2 \\
${ }^{3} P_{1}$ & 2 & 2 & 1 & 2 \\
${ }^{3} P_{2}$ & 3 & 3 & 1 & 2 \\
\hline${ }^{3} P_{2^{-}} F_{2}$ & 2 & 1 & 0 & 1 \\
\hline${ }^{1} D_{2}$ & 2 & 3 & 0 & 1 \\
${ }^{3} D_{1}$ & 2 & 1 & 0 & 1 \\
${ }^{3} D_{2}$ & 2 & 2 & 0 & 1 \\
${ }^{3} D_{3}$ & 1 & 2 & 0 & 1 \\
\hline${ }^{3} D_{3}{ }^{3} G_{3}$ & 1 & 0 & 0 & 0 \\
\hline${ }^{1} F_{3}$ & 1 & 1 & 0 & 0 \\
${ }^{3} F_{2}$ & 1 & 2 & 0 & 0 \\
${ }^{3} F_{3}$ & 1 & 2 & 0 & 0 \\
${ }^{3} F_{4}$ & 2 & 1 & 0 & 0 \\
\hline${ }^{3} F_{4}{ }^{3} H_{4}$ & 0 & 0 & 0 & 0 \\
\hline${ }^{1} G_{4}$ & 1 & 0 & 0 & 0 \\
${ }^{3} G_{3}$ & 0 & 1 & 0 & 0 \\
${ }^{3} G_{4}$ & 0 & 1 & 0 & 0 \\
${ }^{3} G_{5}$ & 0 & 1 & 0 & 0 \\
\hline${ }^{T o t a l}$ & 35 & 38 & 9 & 24 \\
\hline${ }^{2}$ & & &
\end{tabular}

\section{Chiral NN potentials}

The two-nucleon system is non-perturbative as evidenced by the presence of shallow bound states and large scattering lengths. Weinberg [18 showed that the strong enhancement of the scattering amplitude arises from purely nucleonic intermediate states. He therefore suggested to use perturbation theory to calculate the NN potential and to apply this potential in a scattering equation (Lippmann-Schwinger or Schrödinger equation) to obtain the NN amplitude. We follow this philosophy.

Chiral perturbation theory is a low-momentum expansion. It is valid only for momenta $Q \ll \Lambda_{\chi} \approx 1 \mathrm{GeV}$. Therefore, when a potential is constructed, all expressions (contacts and irreducible pion exchanges) are multiplied with a regulator function,

$$
\exp \left[-\left(\frac{p}{\Lambda}\right)^{2 n}-\left(\frac{p^{\prime}}{\Lambda}\right)^{2 n}\right],
$$

where $p$ and $p^{\prime}$ denote, respectively, the magnitudes of the initial and final nucleon momenta in the center-of-mass frame; and $\Lambda \ll \Lambda_{\chi}$. The exponent $2 n$ is to be chosen such that the regulator generates powers which are beyond the order at which the calculation is conducted.

NN potentials based upon $\chi \mathrm{PT}$ at NNLO 22, 29, are poor in quantitative terms; they reproduce the $\mathrm{NN}$ data below $290 \mathrm{MeV}$ lab. energy with a $\chi^{2} /$ datum of more than 20 (cf. tables 3 and 4, below). As shown first by Entem and Machleidt [23], one 
Table 3. $\chi^{2} /$ datum for the reproduction of the $1999 n p$ database below 290 $\mathrm{MeV}$ by various $n p$ potentials. $(\Lambda=500 \mathrm{MeV}$ in all chiral potentials. $)$

\begin{tabular}{cccccc}
\hline Bin $(\mathrm{MeV})$ & \# of data & $\mathrm{N}^{3} \mathrm{LO}^{a}$ & $\mathrm{NNLO}^{b}$ & $\mathrm{NLO}^{b}$ & $\mathrm{AV}^{c} 8^{c}$ \\
\hline $0-100$ & 1058 & 1.06 & 1.71 & 5.20 & 0.95 \\
$100-190$ & 501 & 1.08 & 12.9 & 49.3 & 1.10 \\
$190-290$ & 843 & 1.15 & 19.2 & 68.3 & 1.11 \\
\hline $0-290$ & 2402 & 1.10 & 10.1 & 36.2 & 1.04 \\
\hline
\end{tabular}

${ }^{a}$ Reference [23]. $\quad{ }^{b}$ Reference [29]. $\quad{ }^{c}$ Reference 8].

has to go to order $\mathrm{N}^{3} \mathrm{LO}$ to obtain a $\mathrm{NN}$ potential of acceptable accuracy. Therefore, we will discuss now specifically the NN potential at $\mathrm{N}^{3} \mathrm{LO}$.

At $\mathrm{N}^{3} \mathrm{LO}$, there are 24 contact terms (24 parameters) which contribute to the partial waves with $L \leq 2$. In table 2 , column ' $\mathrm{N}^{3} \mathrm{LO} / Q^{4}$ ', we show how these terms/parameters are distributed over the various partial waves. For comparison, we also show the number of parameters used in the Nijmegen partial wave analysis (PWA93) [4] and in the high-precision CD-Bonn potential [10]. The table reveals that, for $S$ and $P$ waves, the number of parameters used in high-precision phenomenology and in EFT at $\mathrm{N}^{3} \mathrm{LO}$ are about the same. Thus, the EFT approach provides retroactively a justification for what the phenomenologist of the 1990's were doing. At NLO and NNLO 30, the number of parameters is substantially smaller than for PWA93 and CD-Bonn, which explains why this order is insufficient for a quantitative potential.

For an accurate fit of the low-energy $p p$ and $n p$ data, charge-dependence is important. Charge-dependence up to next-to-leading order of the isospin-violation scheme (NL $\varnothing$, in the notation of Ref. 31]) includes: the pion mass difference in OPE and the Coulomb potential in $p p$ scattering, which takes care of the L $\varnothing$ contributions. At order NL $\varnothing$ we have pion mass difference in the NLO part of TPE, $\pi \gamma$ exchange [32, and two charge-dependent contact interactions of order $Q^{0}$ which make possible an accurate fit of the three different ${ }^{1} S_{0}$ scattering lengths, $a_{p p}, a_{n n}$, and $a_{n p}$.

In the optimization procedure, we fit first phase shifts, and then we refine the fit by minimizing the $\chi^{2}$ obtained from a direct comparison with the data. The $\chi^{2} /$ datum for the fit of the $n p$ data below $290 \mathrm{MeV}$ is shown in table 3, and the corresponding one for $p p$ is given in table 4 . The $\chi^{2}$ tables show the quantitative improvement of the $\mathrm{NN}$ interaction order by order in a dramatic way. Even though there is considerable improvement when going from NLO to NNLO, it is clearly seen that $\mathrm{N}^{3} \mathrm{LO}$ is needed

Table 4. $\chi^{2} /$ datum for the reproduction of the $1999 p p$ database below 290 $\mathrm{MeV}$ by various $p p$ potentials. $(\Lambda=500 \mathrm{MeV}$ in all chiral potentials. $)$

\begin{tabular}{cccccc}
\hline Bin $(\mathrm{MeV})$ & \# of data & $\mathrm{N}^{3} \mathrm{LO}^{a}$ & $\mathrm{NNLO}^{b}$ & $\mathrm{NLO}^{b}$ & $\mathrm{AV}^{c} 8^{c}$ \\
\hline $0-100$ & 795 & 1.05 & 6.66 & 57.8 & 0.96 \\
$100-190$ & 411 & 1.50 & 28.3 & 62.0 & 1.31 \\
$190-290$ & 851 & 1.93 & 66.8 & 111.6 & 1.82 \\
\hline $0-290$ & 2057 & 1.50 & 35.4 & 80.1 & 1.38 \\
\hline${ }^{a}$ Reference & 23. & \multicolumn{5}{c}{${ }^{b}$ See footnote } & 34. & \multicolumn{5}{c}{${ }^{c}$ Reference } & 8.
\end{tabular}



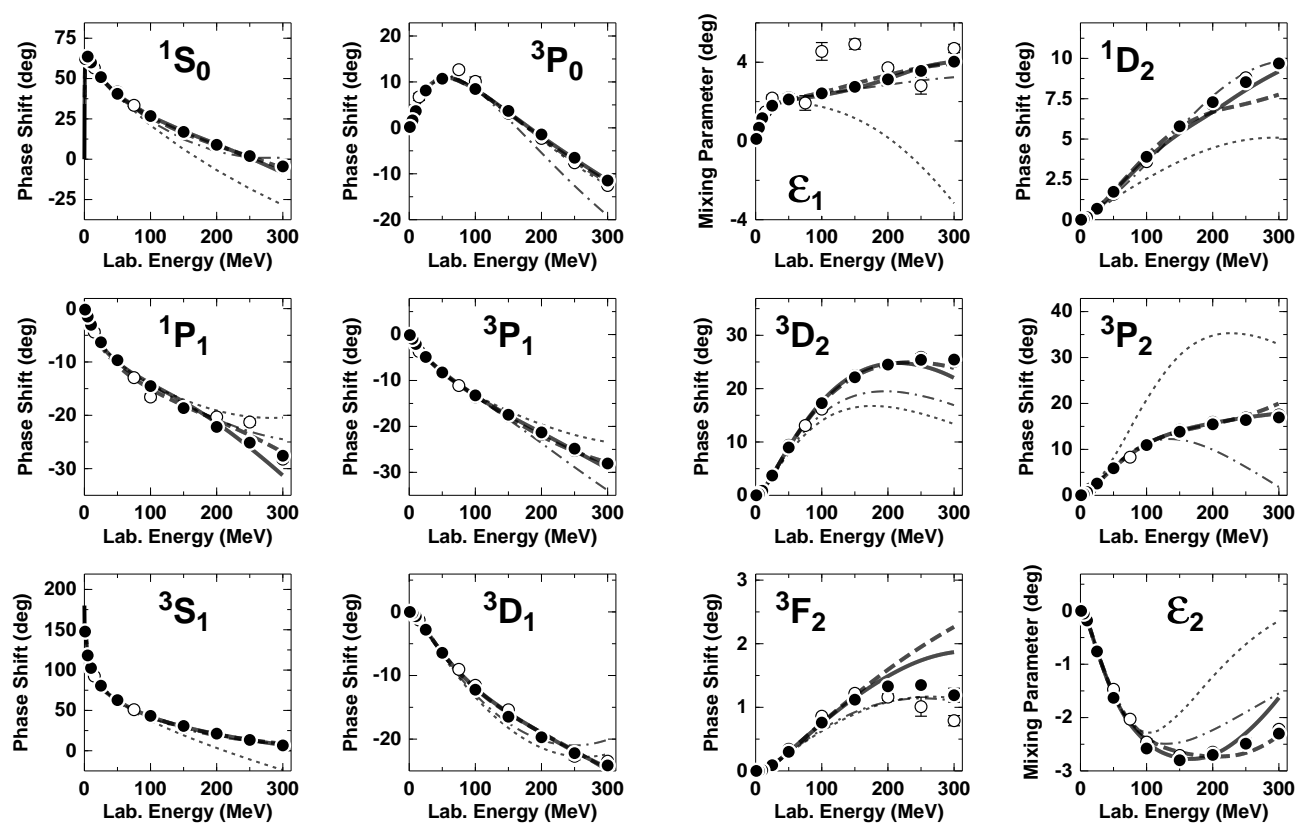

Figure 2. $n p$ phase parameters below $300 \mathrm{MeV}$ lab. energy for partial waves with $J \leq 2$. The thick solid (dashed) line is the result by Entem and Machleidt 23 at $\mathrm{N}^{3} \mathrm{LO}$ using $\Lambda=500 \mathrm{MeV}(\Lambda=600 \mathrm{MeV})$. The thin dotted and dash-dotted lines are the phase shifts at NLO and NNLO, respectively, as obtained by Epelbaum et al. 29] using $\Lambda=500 \mathrm{MeV}$. The solid dots show the Nijmegen multienergy $n p$ phase shift analysis 4, and the open circles are the GWU/VPI single-energy $n p$ analysis SM99 [33.

to achieve an accuracy comparable to the phenomenological high-precision Argonne $V_{18}$ potential [8]. Note that proton-proton data have, in general, smaller errors than $n p$ data which explains why the $p p \chi^{2}$ are always larger.

The phase shifts for $n p$ scattering below $300 \mathrm{MeV}$ lab. energy are displayed in figures 2. What the $\chi^{2}$ tables revealed, can be seen graphically in this figure. The ${ }^{2} P_{2}$ phase shifts are a particularly good example: NLO (dotted line) is clearly poor. NNLO (dash-dotted line) brings improvement and describes the data up to about 100 $\mathrm{MeV}$. The difference between the NLO and NNLO curves is representative for the uncertainty at NLO. and, similarly, the difference between NNLO and $\mathrm{N}^{3} \mathrm{LO}$ reflects the uncertainty at NNLO. Obviously, at $\mathrm{N}^{3} \mathrm{LO}(\Lambda=500 \mathrm{MeV}$, thick solid line $)$ we have a good description up to $300 \mathrm{MeV}$. An idea of the uncertainty at $\mathrm{N}^{3} \mathrm{LO}$ can be obtained by varying the cutoff parameter $\Lambda$. The thick dashed line is $\mathrm{N}^{3} \mathrm{LO}$ using $\Lambda=600 \mathrm{MeV}$. In most cases, the latter two curves are not distinguishable on the scale of the figures. Noticeable differences occur only in ${ }^{1} D_{2},{ }^{3} F_{2}$, and $\epsilon_{2}$ above $200 \mathrm{MeV}$.

\section{Chiral three-nucleon forces}

As noted before, the first non-vanishing 3NF terms occur at NNLO and are shown in figure 1 (row ' $Q 3 / \mathrm{N}^{2} \mathrm{LO}$ ', column ' $3 \mathrm{~N}$ Force'). There are three diagrams: the TPE, 
OPE, and $3 \mathrm{~N}$-contact interactions [28. The TPE $3 \mathrm{~N}$-potential is given by

$$
V_{\mathrm{TPE}}^{3 \mathrm{NF}}=\left(\frac{g_{A}}{2 f_{\pi}}\right)^{2} \frac{1}{2} \sum_{i \neq j \neq k} \frac{\left(\vec{\sigma}_{i} \cdot \vec{q}_{i}\right)\left(\vec{\sigma}_{j} \cdot \vec{q}_{j}\right)}{\left(q_{i}^{2}+m_{\pi}^{2}\right)\left(q_{j}^{2}+m_{\pi}^{2}\right)} F_{i j k}^{\alpha \beta} \tau_{i}^{\alpha} \tau_{j}^{\beta}
$$

with $\vec{q}_{i} \equiv \vec{p}_{i}^{\prime}-\vec{p}_{i}$, where $\vec{p}_{i}$ and $\vec{p}_{i}^{\prime}$ are the initial and final momenta of nucleon $i$, respectively, and

$$
F_{i j k}^{\alpha \beta}=\delta^{\alpha \beta}\left[-\frac{4 c_{1} m_{\pi}^{2}}{f_{\pi}^{2}}+\frac{2 c_{3}}{f_{\pi}^{2}} \vec{q}_{i} \cdot \vec{q}_{j}\right]+\frac{c_{4}}{f_{\pi}^{2}} \sum_{\gamma} \epsilon^{\alpha \beta \gamma} \tau_{k}^{\gamma} \vec{\sigma}_{k} \cdot\left[\vec{q}_{i} \times \vec{q}_{j}\right] .
$$

The vertex involved in this $3 \mathrm{NF}$ term is the two-derivative $\pi \pi N N$ vertex (large solid dot in figure 1) which we encountered already in the TPE contribution to the $2 \mathrm{~N}$ potential at NNLO. Thus, there are no new parameters and the contribution is fixed by the LECs used in NN. The OPE contribution is

$$
V_{\mathrm{OPE}}^{3 \mathrm{NF}}=D \frac{g_{A}}{8 f_{\pi}^{2}} \sum_{i \neq j \neq k} \frac{\vec{\sigma}_{j} \cdot \vec{q}_{j}}{q_{j}^{2}+m_{\pi}^{2}}\left(\boldsymbol{\tau}_{i} \cdot \boldsymbol{\tau}_{j}\right)\left(\vec{\sigma}_{i} \cdot \vec{q}_{j}\right)
$$

and, finally, the $3 \mathrm{~N}$ contact term reads

$$
V_{\mathrm{ct}}^{3 \mathrm{NF}}=E \frac{1}{2} \sum_{j \neq k} \boldsymbol{\tau}_{j} \cdot \boldsymbol{\tau}_{k}
$$

The last two $3 \mathrm{NF}$ terms involve two new vertices (that do not occur in the $2 \mathrm{~N}$ problem), namely, the $\pi N N N N$ vertex with parameter $D$ and a $6 N$ vertex with parameters $E$, To pin them down, one needs two $3 \mathrm{~N}$ observables. In reference [28, the triton binding energy and the $n d$ doublet scattering length ${ }^{2} a_{n d}$ were used. Once $D$ and $E$ are fixed, the results for other $3 \mathrm{~N}, 4 \mathrm{~N}, \ldots$ observables are predictions. In reference [35], encouraging results were reported for ${ }^{6} \mathrm{Li}$. Concerning the famous ' $A_{y}$ puzzle', the above $3 \mathrm{NF}$ terms yield some improvement of the predcited $n d A_{y}$, however, the problem is not resolved.

One should note that there are additional 3NF terms at NNLO due to relativistic corrections $\left(1 / M_{N}\right.$ corrections) that have not yet been included in any calculation. However, there are all reasons to believe that these contributions will be very small, probably negligible. It is more likely that the problem with the chiral $3 \mathrm{NF}$ is analogous to the one with the chiral 2NF: namely, NNLO is insufficient and for sufficient accuracy one has to proceed to $\mathrm{N}^{3} \mathrm{LO}$. Two $3 \mathrm{NF}$ terms at $\mathrm{N}^{3} \mathrm{LO}$ are indicated in figure 1 . The $\mathrm{N}^{3} \mathrm{LO} 3 \mathrm{NF}$, which will probably not depend on any new parameters, is presently under development.

\section{Conclusions}

The EFT approach to nuclear forces is superior to all earlier nuclear force models. It represents a scheme that has an intimate relationship with QCD and allows to calculate nuclear forces to any desired accuracy. Moreover, nuclear two- and many-body forces are generated on the same footing.

At $\mathrm{N}^{3} \mathrm{LO}$ [23], the accuracy is achieved that is necessary and sufficient for reliable nuclear structure calculations. First calculations applying the $\mathrm{N}^{3} \mathrm{LO}$ potential have produced promising results [35, 36, 37, 38, 39, 40, 41].

The 3NF at NNLO is known [28] and has had first successful applications [35]. The $3 \mathrm{NF}$ and $4 \mathrm{NF}$ at $\mathrm{N}^{3} \mathrm{LO}$ is presently under construction.

In summary, the stage is set for many years of exciting nuclear structure research that is more consistent than anything we had before. 


\section{Acknowledgments}

This work was supported by the U.S. National Science Foundation under Grant No. PHY-0099444, by the Spanish Ministerio de Ciencia y Tecnología under Contract No. BFM2001-3563, and the Junta de Castilla y León under Contract No. SA-104/04.

\section{References}

[1] Kukulin V I et al. 2002 Int. J. Mod. Phys. E 111

[2] Doleschall P et al. 2003 Phys. Rev. C 67064005

[3] Shirokov A et al. 2004 arXiv:nucl-th/0407018 and contribution to this workshop

[4] Stoks V G J, Klomp R A M, Rentmeester M C M and de Swart J J 1993 Phys. Rev. C 48792

[5] Lacombe M et al. 1980 Phys. Rev. C 21861

[6] Machleidt R, Holinde K, and Elster C 1987 Phys. Rep. 1491

[7] Stoks V G J, Klomp R A M, Terheggen C P F and de Swart J J 1994 Phys. Rev. C 492950

[8] Wiringa R B, Stoks V G J and Schiavilla R 1995 Phys. Rev. C 5138

[9] Machleidt R, Sammarruca F and Song Y 1996 Phys. Rev. C 531483

[10] Machleidt R 2001 Phys. Rev. C 63024001

[11] Nagels M M, Rijken T A and de Swart J J 1978 Phys. Rev. D 17768

[12] Reid R V 1968 Ann. Phys. (N.Y.) 50411

[13] Glöckle W et al 1996 Phys. Rep. 274107

[14] Engvik L, Hjorth-Jensen M, Machleidt R, Muether H, and Polls A 1997 Nucl. Phys. A627 85

[15] Polls A, Muether H, Machleidt R, and Hjorth-Jensen M 1998 Phys. Lett. B432 1

[16] Caurier E, Navrátil P, Ormand W E, and Vary J P 2002 Phys. Rev. C 66024314

[17] Machleidt R 1989 Adv. Nucl. Phys. 19189

[18] Weinberg S 1991 Nucl. Phys. B363 3

[19] Ordóñez C, Ray L and van Kolck U 1996 Phys. Rev. C 532086

[20] van Kolck U 1999 Prog. Part. Nucl. Phys. 43337

[21] Kaiser N et al. 1997 Nucl. Phys. A625 758

[22] Epelbaum E et al. 2000 Nucl. Phys. A671 295

[23] Entem D R and Machleidt R 2003 Phys. Rev. C 68041001

[24] Kaiser N 2001 Phys. Rev. C 64057001 Kaiser N 2002 Phys. Rev. C 65017001

[25] Entem D R and Machleidt R 2002 Phys. Rev. C 66014002

[26] Kaiser N 1999 Phys. Rev. C 61014003

Kaiser N 2000 Phys. Rev. C 62024001

[27] van Kolck U 1994 Phys. Rev. C 492932

[28] Epelbaum E et al. 2002 Phys. Rev. C 66064001

[29] Epelbaum E et al. 2002 Eur. Phys. J. A15 543

[30] Note that, due to parity, contact terms appear only at even powers of $Q$ which is why the contacts are the same for NLO and NNLO.

[31] Walzl M et al. 2001 Nucl. Phys. A693 663

[32] van Kolck U et al. 1998 Phys. Rev. Lett. 804386

[33] SAID, solution SM99 (Summer 1999).

[34] Since Ref. 29] provides only the $n p$ versions of the NLO and NNLO potentials, we have constructed the $p p$ versions by incorporating charge-dependence and minimizing the $p p \chi^{2}$.

[35] Nogga A et al. 2004 Nucl. Phys. A737 236

[36] Kowalski K, Dean D J, Hjorth-Jensen M and Papenbrock T 2004 Phys. Rev. Lett. 92132501

[37] Dean D J and Hjorth-Jensen M 2004 Phys. Rev. C 69054320

[38] Wloch $\mathrm{M}$ et al. 2005 arXiv:nucl-th/0501067

[39] Dean D J et al., contribution to this workshop

[40] Navrátil P and Caurier E 2003 arXiv:nucl-th/0311036

[41] Forssen C, Navrátil P, Ormand W E and Caurier E 2004 arXiv:nucl-th/0412049 